\title{
Subsonic Wing Planform Design Using Multidisciplinary Optimization
}

\author{
Sean Wakayama* and Ilan Kroot \\ Stanford University, Stanford, California 94305
}

\begin{abstract}
This article presents basic results from wing planform optimization for minimum drag with constraints on structural weight and maximum lift. Analyses in each of these disciplines are developed and integrated to yield successful optimization of wing planform shape. Results demonstrate the importance of weight constraints, compressibility drag, maximum lift, and static aeroelasticity on wing shape, and the necessity of modeling these effects to achieve realistic optimized planforms.
\end{abstract}

\section{Nomenclature}

$A, \quad=$ rib boom cross-sectional area

$A_{c} \quad=$ area enclosed by structural box cross section

$A_{\mathrm{w}} \quad=$ rib web cross-sectional area

$b \quad=$ span

$b_{s} \quad=$ stringer spacing

$C_{L} \quad=$ wing lift coefficient

$C_{L} \quad=$ wing maximum lift coefficient

$\mathrm{C}^{L_{\text {mitix }}}=$ chord

$c_{c,} \quad=$ wing box chord

$c_{d} \quad=$ section drag coefficient

$c_{l} \quad=$ section lift coefficient

$c_{l} \quad=$ maximum allowable section lift coefficient

$E^{\text {mix }}=$ Young's modulus

$G \quad=$ shear modulus

I $=$ section bending inertia

$I_{\mathrm{ref}}=$ dimensionless skin bending inertia

$J \quad=$ section torsional inertia

$K, \quad=$ local buckling coefficient

$L \quad=$ rib spacing

$\hat{\boldsymbol{n}} \quad=$ local panel unit normal vector

$P \quad=$ bending end load $=$ bending stress times smeared skin thickness

$p_{a} \quad=$ surface pressure

$q \quad=$ dynamic pressure

$\mathrm{S} \quad=$ wing area

$s \quad=$ line integral coordinate

$T_{\text {ret }} \quad=$ ratio of smeared and actual skin thickness

$t=$ wing thickness

$t_{3} \quad=$ skin thickness

$x, y, z=$ coordinate directions, nominally along freestream, pointing to aircraft right and pointing up, respectively

$z_{\mathrm{cr}}=$ dimensionless height location of critical stringer stress

$\varepsilon \quad=$ jig twist

$\eta \quad=$ coordinate direction parallel to elastic axis of wing

A $=$ sweep

$\Lambda_{\text {c:a }}=$ sweep of the elastic axis

$\checkmark=$ Poisson's ratio

Received July 28, 1994; revision received Dec. 24, 1994; accepted for publication Dec. 28, 1994. Copyright (C) 1995 by S. Wakayama and I. Kroo. Published by the American Institute of Aeronautics and Astronautics, Inc., with permission.

'Graduate Student, Department of Aeronautics and Astronautics. Student Member AIAA.

$¥$ Associate Professor, Department of Aeronautics and Astronautics. Member AIAA. $\xi=$ coordinate direction perpendicular to elastic axis of wing

$P \quad=$ density

$\rho_{\text {mat }}=$ structural material density

$\sigma_{v} \quad=$ yield stress

$\phi \quad=$ dihedral angle

\section{Introduction}

O PTIMIZATION can be a powerful tool in the conceptual and preliminary phases of wing design; however, successful planform optimization has been an elusive goal, with the imposition of designer expertise often necessary to avoid unrealistic results. This lack of success comes from the high sensitivity of wing shape to off-design considerations. By casting these considerations as constraints and selecting a reasonable performance objective, realistic planforms can be attained; the problem is then to build an optimization analysis that appropriately models the aerodynamic and structural design drivers.

Different levels of analysis have been used for wing optimization, ranging from simple analytic or empirical expressions for conceptual design,' to complex finite element structural models.' The difficulty is to find or develop analyses that are sufficiently simple to be called thousands of times during optimization, but are sophisticated enough to capture considerations that determine local geometry.

With a simplified objective, such as minimum-induced and section profile drag with structural weight constraints, the optimization can be cast as a linear problem that can be solved directly to obtain optimal load distributions for given planf o r m .Sdme extension of this can be made toward determining chord or thickness distributions. ${ }^{5,6}$ These methods are very fast, but cannot be extended to planform variables such as sweep, span, or dihedral.

Nonlinear optimization provides the flexibility for planform design and is used in Refs. 2 and 7-9. Reference 8 uses analyses that are similar to those used in this article, but for an aircraft sizing problem rather than the more detailed wing design problem treated here.

This article describes extensions to the aerodynamic and structural analyses used in Ref. 9 and presents new optimization results. Complete descriptions and validation of the analyses are made in Ref. 10. This forms a methodology for planform optimization of subsonic wings.

Basic results of this method are presented here, demonstrating wing characteristics driven by many considerations, including induced, profile, and compressibility drag, bending and buckling weight, section maximum lift constraints, and static aeroelasticity. The results strongly indicate that drag, weight, and maximum lift must be carefully analyzed to obtain reasonable planform shapes. 


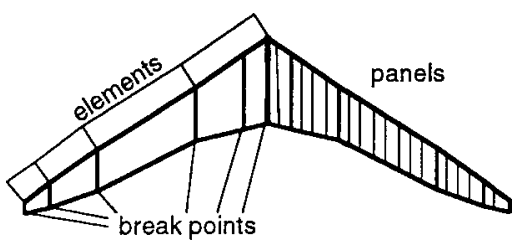

Fig. 1 Wing element and panel geometry.

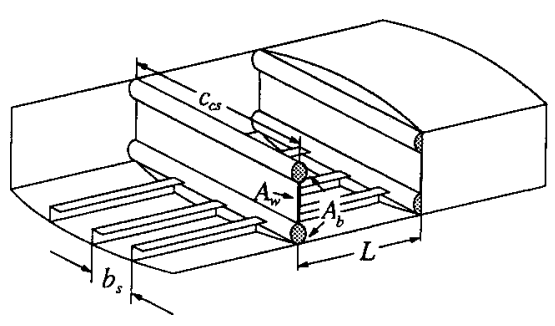

Fig. 2 Structural geometry.

\section{Geometry}

Wings are modeled with a set of lifting surface elements, shown in Fig. 1, described by the following parameters: span, area, sweep, dihedral, and taper ratio. To provide the discretization for both aerodynamic and structural models, elements are divided into panels. Twist and thickness-to-chord ratios are specified at break points. Break points usually lie on element root and tip edges, but may be selected to lie between any two panels in an element. Twist and wing thickness are varied linearly between break points. This scheme provides a concise set of variables for optimization.

\section{Structural Modeling}

The structural model has two levels of detail. The first, drawn in Fig. 2, is used for calculating skin thicknesses and weight. Skin panels are described with skin thickness $t_{s}$, stringer spacing $b$, and rib spacing $\boldsymbol{L}$. The stringer geometry is idealized as scaling with $\boldsymbol{b}$, and $t$,. This allows the stringer geometry to be represented with three parameters, $I_{\text {rcf }}, z_{\text {сr }}$ and $T_{\text {ref }}$ : where $I_{\mathrm{rct}} b_{*}^{2} t_{\mathrm{s}}=$ panel bending inertia/unit chordwise length, $z_{\mathrm{cr}} b_{\mathrm{s}}=$ height location of maximum stress, and $T_{\text {ret }}=$ smeared skin thickness/actual skin thickness.

This representation was chosen to facilitate analytic expressions for best rib and stringer spacings that are varied along the span; it does not represent a practical design. For manufacturing ease, fixed stringer cross sections and spacings are used. Actual stringer designs should be close to optimal in the heaviest sections of a given wing, however, so that the weights estimated in the following method are reasonable. The rib cross section is modeled as a shear web capped with two booms to take up bending. The web area is $\boldsymbol{A}$,, and the combined boom area is $\boldsymbol{A}$,.

The second level in the structural model treats the wing as a thin-walled, single-cell monocoque beam and is used to calculate stiffness. The beam cross section has vertical fore and aft spars, parabolic upper and lower skins, and a constant skin thickness around the section. Stringer and spar cap material are combined with actual skin material to obtain an effective smeared skin thickness that is used for bending stiffness calculations.

Structural properties are calculated using methods described in Refs. 10 and 11. Bending inertias are calculated in the standard manner. Torsional inertias are computed with Bredt-Batho theory. Stringer area is included in the smeared skin thickness for calculating bending stiffness, but not for torsional stiffness, where it gives little practical contribution.

The $x$ coordinate of the shear center is calculated for sections along the span of the wing using standard methods. ${ }^{10,11}$
The line of shear center locations then defines the elastic axis sweep $\Lambda_{\text {ca }}$, which is needed for calculating aeroelastic loads.

\section{Aeroelastic Lift Distribution}

A method similar to that described in Ref. 12 is used to evaluate lift distributions on flexible wings. The wing is represented by a set of discrete horseshoe vortices with bound vortices located at the quarter chord. Vortex strengths are determined by enforcing tangent flow boundary conditions at control points located at the three-quarter chord:

$$
\boldsymbol{U}_{\infty} \cdot \boldsymbol{n}_{i}+\sum_{j} \boldsymbol{V}_{\mathrm{ind}_{i j}} \cdot \hat{\boldsymbol{n}}_{i}=0
$$

$\hat{n}_{i}$ is the unit vector for panel i. $\boldsymbol{V}_{\text {ind }_{i i}}$ is the velocity induced at panel i due to the vortex $j$; it is calculated by integrating velocity contributions over vortex $j$ using the Biot-Savart law. $\boldsymbol{V}_{\text {ind }}$ is proportional to the strength of vortex $j$, yielding the following equation:

$$
\sum_{j} \mathrm{AIC}_{i j} \bar{\Gamma}_{j}=-\hat{U}_{x} \cdot \hat{n}_{i}
$$

or in matrix form

$$
[\mathrm{AIC}]\{\widetilde{\Gamma}\}=\{b c\}
$$

The aerodynamic influence coefficient matrix, [AIC], relates the vortex strengths to the normal velocities induced at the control points. The boundary condition vector $\{\mathbf{b c}\}$ denotes the flow through the control points due to the freestream. $\boldsymbol{U}_{x}$ denotes a unit vector in the freestream direction, and $\Gamma$ represents the actual vortex strength normalized by the freestream velocity.

Moments, needed to calculate elastic deformations and size skin thicknesses, are evaluated to first-order by

$$
\boldsymbol{M}_{i}=\sum_{i} 2 q \operatorname{iconn}(j, i)\left[\boldsymbol{r}_{i j} \times\left(\hat{\boldsymbol{U}}_{\infty} \times \boldsymbol{G}\right)\right] \overline{\boldsymbol{\Gamma}}_{j}
$$

The vector $\boldsymbol{r}_{i j}$ points from the shear center of panel i to the bound vortex center of panel $j$. The function, iconn $(j, i)$, has a value of 1 when loads on $j$ logically affect moments on $i$; it has a value of 0 when loads on $j$ have no influence, as when panel $j$ lies inboard of panel i. The vector $\boldsymbol{G}$ runs from root to tip of bound vortex $j$.

The moment vector is separated into bending and torsional components about the elastic axis:

$$
\begin{aligned}
\left\{M_{\text {tend }}\right\} & =q\left[\mathrm{MIC}_{\text {tend }}\right]\{\bar{\Gamma}\} \\
\left\{M_{\text {torss }}\right\} & =q\left[\mathrm{MIC}_{\text {tors }}\right]\{\bar{\Gamma}\}
\end{aligned}
$$

Integrating these moments along the span yields elastic slope and twist deformations:

$$
\begin{gathered}
\frac{\mathrm{d} w}{\mathrm{~d} \eta}(\eta)=\int_{0}^{\eta}\left[\hat{\boldsymbol{\xi}}(\eta) \cdot \hat{\boldsymbol{\xi}}(s) \frac{M_{\text {bend }}}{E I}+\hat{\boldsymbol{\xi}}(\eta) \cdot \hat{\boldsymbol{\eta}}(s) \frac{M_{\text {tors }}}{G J}\right] \mathrm{d} s \\
\theta(\eta)=\int_{0}^{\eta}\left[\hat{\boldsymbol{\eta}}(\eta) \cdot \hat{\boldsymbol{\xi}}(s) \frac{M_{\text {bend }}}{E I}+\hat{\boldsymbol{\eta}}(\eta) \cdot \hat{\boldsymbol{\eta}}(s) \frac{M_{\text {tors }}}{G J}\right] \mathrm{d} s
\end{gathered}
$$

Where $\hat{\boldsymbol{\xi}}$ and $\hat{\boldsymbol{\eta}}$ are unit vectors aligned, respectively, perpendicular to and parallel to the local elastic axis of the wing structure.

These deformations cause rotation of the panel incidences:

$$
A=A_{\prime \prime \prime}+\frac{\mathrm{dw}}{\mathrm{d} \eta} \hat{\boldsymbol{\xi}} \times A_{\prime \prime \prime}+\theta \hat{\boldsymbol{\eta}} \times A_{\prime \prime \prime}
$$


Through the integration and rotation above, $\hat{\boldsymbol{n}}$ is linearly related to the vortex strengths. Recalling that $\{b c\}=\boldsymbol{U}_{\infty} \cdot \hat{\boldsymbol{n}}$, we define the following:

$$
\begin{aligned}
& K_{\text {bend }_{i j}}=\hat{U}_{\infty} \cdot\left\{\left[\left(\hat{\boldsymbol{\xi}}_{i} \cdot \hat{\boldsymbol{\xi}}_{j}\right) \hat{\boldsymbol{\xi}}_{i}+\left(\hat{\boldsymbol{\eta}}_{i} \cdot \hat{\boldsymbol{\xi}}_{j}\right) \hat{\boldsymbol{\eta}}_{i}\right] \times \hat{\boldsymbol{n}}_{\mathrm{jig}}\right\} \\
& K_{\text {tors }_{i j}}=\hat{U}_{\infty} \cdot\left\{\left[\left(\hat{\boldsymbol{\xi}}_{i} \cdot \hat{\boldsymbol{\eta}}_{j}\right) \hat{\boldsymbol{\xi}}_{i}+\left(\hat{\boldsymbol{\eta}}_{i} \cdot \hat{\boldsymbol{\eta}}_{j}\right) \hat{\boldsymbol{\eta}}_{i}\right] \times \hat{\boldsymbol{n}}_{\mathrm{jig}}\right\}
\end{aligned}
$$

The aeroelastic boundary condition is then

$$
\{b c\}=\left\{b c_{\mathrm{jig}}\right\}+\left\{\Delta b c_{\text {elastic }}\right\}
$$

where

$$
\begin{aligned}
& \Delta b c_{\text {clastic }_{i}}=q \sum_{j} K_{\text {bend }_{i j}} \operatorname{iconn}(i, j) \frac{\delta \eta_{j}}{E I_{j}} \sum_{k} \operatorname{MIC}_{\text {bend }_{j k}} \bar{\Gamma}_{k} \\
& \quad+q \sum_{j} K_{\operatorname{tor}_{i j}} \operatorname{iconn}(i, j) \frac{\delta \eta_{j}}{G J} \sum_{k} \operatorname{MIC}_{\text {tors }_{j k}} \bar{\Gamma}_{k}
\end{aligned}
$$

or

$$
\left\{\Delta b c_{\text {elastic }}\right\}=-[\mathrm{SIC}]\{\bar{\Gamma}\}
$$

Recalling the relationship $[\mathrm{AIC}]\{\bar{\Gamma}\}=\{b c\}$, the equation for determining the vorticity distribution with aeroelastic deformation is then

$$
[\mathrm{AIC}]\{\bar{\Gamma}\}+[\mathrm{SIC}]\{\bar{\Gamma}\}=\left\{b c_{\mathrm{jig}}\right\}
$$

\section{Refinement to Aeroelastic Loads}

While developing the method for obtaining wing weight, it was found that zero-lift pitching moment and inertial bending relief had small but noticeable effects when estimating wing weights of existing transports. These effects are modeled by altering the boundary condition vector.

Moments from zero-lift pitching moments are calculated using

$$
\boldsymbol{M}_{0_{i}}=\sum_{j} q \cos \Lambda c_{m_{0 j}} c_{j}^{2} \operatorname{iconn}(j, i)\left(G_{y_{j}} \hat{\mathbf{y}}+G_{z j} \hat{z}\right)
$$

The cos $\mathbf{A}$ accounts for simple sweep effects and $c_{m_{11}}$ represents the pitching moment for the streamwise airfoil section; $c_{m_{0}}=$ -0.07 for the examples in this article. $G_{y}$ and $G_{z}$ express $y$ and $z$ components of the panel width.

Inertial bending relief is handled simply:

$$
M_{M_{i}}=\sum_{J}-n g m_{j} \operatorname{iconn}(j, i) r_{m_{i j}} \times \hat{z}
$$

Here, $g$ is the acceleration of gravity, $n$ is the load factor, $m_{j}$ is the mass at panel $j$, and $\boldsymbol{r}_{m_{i j}}$ is the vector from shear center of panel $i$ to the shear center of panel $j$. The panel mass $m_{j}$ includes mass of the wing structure, fuel contained in the wing, and any engines mounted at the panel. Fuel mass is distributed evenly through the volume enclosed by the structural box, with the occupied fraction of the volume adjusted to match actual or required mass.

\section{Load Cases}

Structural weight is evaluated using as many as six loading conditions. All flight loading conditions are evaluated at a specified structural design altitude and Mach number. Four of these are done at maximum weight with zero wing fuel: cruise, maneuver, vertical, and lateral gust. A separate maneuver condition is evaluated for the aircraft at maximum takeoff weight with full wing fuel. Maneuver load factor and gust velocities are specified as per Federal Aviation Regulations (FAR) Part 25. A gust alleviation factor is applied to vertical gust velocities. The final loading condition is a taxi bump in which the wing, with full wing fuel, must sustain a 1.5-g vertical acceleration.

For the optimization results in this article, the maneuver condition with full wing fuel is critical. Although the wing receives bending relief from the fuel inertia, the fuel is normally distributed close to the wing root, making the inertia relief less than the bending moments due to the extra load. The other conditions were examined, but were not critical. The zero wing fuel case can be critical because of the lack of bending relief. Gust conditions are evaluated at zero fuel weight, where gust load factors are likely to exceed maneuver load factors. The taxi bump was added when, in an earlier study, the optimizer began to create wingtip tanks to maximize inertia relief. In ground handling, without counteracting air loads, the weight of tip tanks can generate moments in excess of the flight loads, and the taxi condition is used to properly penalize such designs.

\section{Structural Design Constraints}

Several constraints are considered in estimating the weight and stiffness of the wing box.

Given an end load from wing bending $P$ and a specified pressure load $p_{a}$, the skin-stringer panels supported between ribs must not yield:

Panel stress-normal stress

$$
t_{s} \geq \frac{p_{a} L^{2} z_{\mathrm{cr}}}{8 I_{\mathrm{ref}} b_{s} \sigma_{y}} K_{\mathrm{PS}}+\frac{P}{\sigma_{y} T_{\mathrm{ref}}} K_{\mathrm{NS}}
$$

All $K$ parameters, $K,, \quad K$,» etc., are constants for correlating the design equations with actual structures.

Buckling of the skin between stringers is handled in a local buckling constraint.

Local buckling

$$
t_{s} \geq\left[\frac{12\left(1-v^{2}\right) P b_{s}^{2}}{\pi^{2} K_{s} E T_{\mathrm{ref}}}\right]^{1 / 3} K_{\mathrm{LB}}
$$

The buckling of skin-stringer panels between ribs is also considered.

Panel buckling

$$
t_{s} \geq \frac{P L^{2}}{\pi^{2} E I_{\mathrm{ref}} b_{s}^{2} T_{\mathrm{ref}}} K_{\mathrm{PB}}
$$

The major constraint affecting rib sizing is the stiffness required to force panel buckling to occur in cells dictated by the rib spacing and not in lower energy modes involving deflection of the ribs. The rib boom area to do this is given by

Boom buckling

$$
A, \geq \frac{5 P c_{c}^{4}}{48 E t^{2} L} K_{\mathrm{BB}}
$$

\section{Estimates for Minimum Weight Geometry}

Using the given constraint equations, it is possible to analytically determine a geometry that will approximate a minimal weight skin, stringer, and rib structure. These analytic expressions provide rapid structural sizing estimates. The procedure is to pick sets of constraints that will be active, describe rib and stringer spacings as constrained functions of the skin thickness, express the structural weight per unit area as a function only of the skin thickness, and derive an expression for the skin thickness minimizing $(\mathrm{m} / \mathrm{S})$.

To size the structure, optimal skin thicknesses are evaluated for possible combinations of constraints plus minimum gauge. 
The largest skin thickness is taken to be critical and is applied to the constraint equations to determine $b$, and $L$. Equations for the two critical constraint combinations are given below. Additional combinations are discussed in Ref. 10.

Skin sizing for the case where local, panel, and boom buckling constraints are active implies

$$
\begin{gathered}
b_{s}^{2}=\frac{\pi^{2} K_{s} E T_{\mathrm{rcl}} t_{s}^{3}}{12\left(1-\nu^{2}\right) P K_{\mathrm{LB}}^{3}} \\
L^{2}=\frac{\pi^{4} K_{s} E^{2} I_{\mathrm{ref}} T_{\mathrm{ret}}^{2} t_{s}^{4}}{12\left(1-\nu^{2}\right) P^{2} K_{\mathrm{LB}}^{3} K_{\mathrm{PB}}} \\
\frac{(m / S)}{\rho_{\text {mat }}}=2 T_{\mathrm{ref}} t_{s}+\frac{5\left(1-\nu^{2}\right) P^{3} c_{c s}^{4} K_{\mathrm{LB}}^{3} K_{\mathrm{PB}} K_{\mathrm{BB}}}{4 \pi^{4} K_{s} E^{3} I_{\mathrm{ref}} T_{\mathrm{ref}}^{2} t^{2} t_{s}^{4}}+\frac{A_{w}}{L} \\
t_{s}^{5}=\frac{5\left(1-\nu^{2}\right) P^{3} c_{c s}^{4} K_{\mathrm{LB}}^{3} K_{\mathrm{PB}} K_{\mathrm{BB}}}{2 \pi^{4} K_{s} E^{3} I_{\mathrm{ref}} T_{\mathrm{ref}}^{3} t^{2}}
\end{gathered}
$$

For the case where panel and normal stress, local and boom buckling constraints are active, the sizing algorithm is more complicated. We define two variables:

$$
\begin{aligned}
t_{s_{2}} & =\frac{p_{a} L^{2} z_{\mathrm{cr}}}{8 I_{\mathrm{ref}} b_{s} \sigma_{y}} K_{\mathrm{PS}} \\
t_{s_{3}} & =\frac{P}{\sigma_{y} T_{\mathrm{ref}}} K_{\mathrm{NS}}
\end{aligned}
$$

The required skin thickness is

$$
t_{s}=t_{s_{2}}+t_{s_{3}}
$$

The equations for sizing the skin structure are

$$
\begin{aligned}
& b_{s}^{2}=\frac{\pi^{2} K_{s} E T_{\mathrm{ref}}}{12\left(1-\nu^{2}\right) P} \frac{\left(t_{s_{2}}+t_{s s_{3}}\right)^{3}}{K_{\mathrm{LB}}^{3}} \\
& L^{2}=\frac{8 I_{\mathrm{rct}} \sigma_{\mathrm{r}}}{p_{\mathrm{u}} z_{\mathrm{cr}}} b, \frac{t_{\mathrm{s}}}{K_{\mathrm{PS}}} \\
& \frac{(m / S)}{\rho_{\mathrm{mat}}}=2 T_{\mathrm{ref}}\left[\left(t_{s_{2}}+t_{s_{3}}\right)+\frac{T}{\left(t_{s,}+t_{s_{3}}\right)^{3 / 2} t_{s s}}\right]+\frac{A_{n}}{L}
\end{aligned}
$$

where

$$
T=\frac{5 P p_{a} c_{c s}^{4} z_{\mathrm{cr}} K_{\mathrm{PS}} K_{\mathrm{BB}}}{192 E I_{\mathrm{ret}} \sigma_{1} t^{2}\left(2 T_{\mathrm{red}}\right)} \sqrt{\frac{3\left(1-\nu^{2}\right) P K_{\mathrm{LE}}^{3}}{\pi^{2} K E T_{\mathrm{rer}}}}
$$

Minimizing with respect to $t_{s}$ yields the following equation that can be quickly solved using Newton's method on the range $t_{s},>0$ :

$$
\left(t_{s_{2}}+t_{s_{3}}\right)^{5 / 2} t_{s_{2}}^{2}-T\left(\frac{5}{2} t_{s_{2}}+t_{s_{3}}\right)=0
$$

\section{Estimates for Leading- and Trailing-Edge Weight}

Leading and trailing edges transmit pressure loads into the wing box and are too light to carry wing bending loads. They are sized according to the following constraints. The skin should not yield under a specified pressure load. For bending of the skin between ribs, this requires

Local stress

$$
t, \geq \sqrt{\left(3 p_{t} L^{2} / 4 \sigma_{v}\right)} K_{\mathrm{LS}}
$$

The ribs must transmit the pressure loads to the wing spars. The shear web sized by this requirement has area

Web stress

$$
A_{w} \geq\left(\sqrt{3} p_{a} c_{c} L / \sigma_{v}\right) K_{\mathrm{wS}}
$$

The required boom area to support bending stresses is

Boom stress

$$
A_{b} \geq\left(p_{a} c_{e}^{2} L / \sigma_{y} t_{e}\right) K_{\mathrm{BS}}
$$

Here, $c_{c}$ is the edge chord length: the distance from the spar to the end of the airfoil section. $t_{e}$ is the edge thickness: the height of the airfoil section at the spar.

Since $\mathrm{rib}(\mathrm{m} / \mathrm{S})$ is not dependent on $L$, skin $(\mathrm{m} / \mathrm{S})$ suggests $L$ goes to zero. The structure is then sized by minimum gauge with $L$ just large enough to make the local stress constraint critical.

\section{Weight Prediction Details}

The parameters used in the weight method are described here briefly. Additional descriptions may be found in Ref. 10 .

Bending end load $P$ is computed directly from the wing bending moments:

$$
P=\left(M_{\text {bend }} z_{\text {crii }} / I\right) \mathrm{SF} T_{\text {ref }} t_{s}
$$

Here, $z_{\text {crit }}$ is the maximum vertical separation of the skin from the section centroid of area, and SF is the load safety factor $\mathrm{SF}=1.5$

The design skin pressure $p_{a}$ is based upon average lift pressures over representative wing sections. The following form is used to estimate $p_{i}$ :

$$
p_{a}=K_{p_{a}} n_{\text {crit }}\left(W_{A / C} / S\right) S F
$$

$\left(W_{A / C} / S\right)$ is the 1-g wing loading. $n_{\text {crit }}$ is the load factor for the condition setting the skin pressure, and $K_{p_{a}}$ is a correction constant obtained from analysis or empirical data.

The mass of skin, spars, and stringers in the wing box is

$$
m_{\mathrm{skin}}=K_{\mathrm{CS}} \int \rho_{\mathrm{mat}} P_{s} T_{\mathrm{ret}} t_{s} \mathrm{~d} b
$$

Here, $P_{s}$ is the box perimeter, db is the incremental span, and $K_{C S}$ is a constant correction factor, converting our idealized mass to an estimate of actual mass. The mass of ribs in the wing box is

$$
m_{\mathrm{rib}}=K_{\mathrm{CS}} \int \rho_{\mathrm{mat}} \frac{A_{w}+A_{b}}{L} c_{c \mathrm{~s}} \mathrm{~d} b
$$

The mass of leading or trailing edges is

$$
m_{\text {edge }}=K_{\text {edge }} \mid \rho_{\text {mat }}\left(2 T_{\mathrm{ref}} t_{s}+\frac{A_{w}+A_{b}}{\boldsymbol{T}} \quad c_{e} \mathrm{~d} b\right.
$$

Here, $K_{\text {cdge }}=K$, or $K_{\mathrm{TE}}$ is a correction factor similar to $K_{\mathrm{CS}}$. The mass of other items, including joints, doors, wing fuselage attachment, fuel boundaries, landing gear support, fairings, and fillets is assumed to be proportional to the maximum wing loading:

$$
m_{\text {other }} / S=K_{\text {other }}\left(W_{A / C} / S\right) n_{\text {lim }} / g
$$

In a weight study done to correlate the method against actual weights of commercial aircraft wings, the following parameters were found to provide reasonable agreement with actual weights." $K_{\mathrm{LS}}=K_{\mathrm{PS}}=K_{\mathrm{NS}}=K, \quad=K_{\mathrm{PB}}=K_{\mathrm{WS}}=$ 


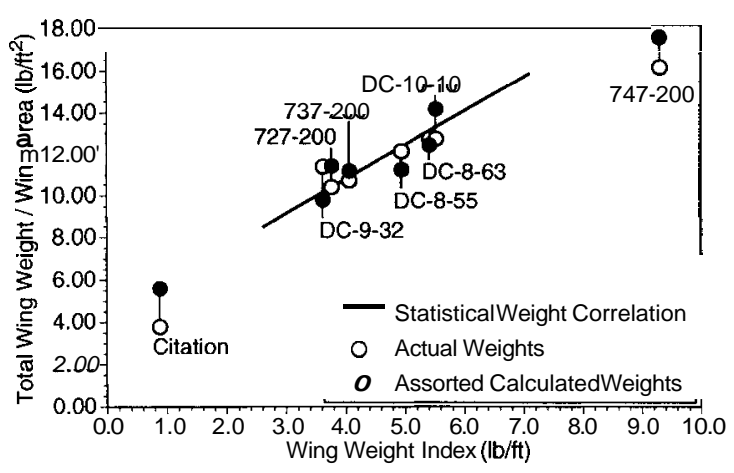

Fig. 3 Calculated wing weights compared against a statistical method and actual weights.

1 , no correction for nonoptimal skin or stringer material. $K_{\mathrm{BS}}$ $=K_{\mathrm{BB}}=3$, equivalent to spreading the area concentrated in the rib booms evenly over the height of the rib web. Corrections for nonoptimal and nonstructural weight were $K_{\mathrm{CS}}$ $=1.2, K_{\mathrm{LE}}=1.6, K$,. $=2.7$, and $K_{\text {other }}=1.1 \times 10^{-2}$. Skin pressure estimates used $K_{p_{a}}=0.83$ and $n_{\text {crit }}=n_{\text {lim }}$ for structural box center sections, $K_{p_{u}}=2.95$ and $n_{\text {crit }}=n_{\text {lim }}$ for leading edges, and $K_{p_{u}}=3.0$ and $n_{\text {crit }}=1.0$ for trailing edges. Dimensionless stringer geometry was taken as $I_{\text {ref }}=1.40325$ $\times 10^{-2}, T_{\text {ret }}=1.69, z_{\text {сг }}=0.25740, K=5.236$. Material properties were $E=10.5 \times 10^{6} \mathrm{psi}, \mathrm{G}=4.0 \times 10^{6} \mathrm{psi}, v$ $=0.34, \sigma_{y}=50 \times 10^{3}$ psi.

Figure 3 shows the method provides reasonable weight estimates when compared to actual weights and weights from a statistical method given in Ref. 13. The weight index is given by

$$
\text { weight index }=\frac{n_{\mathrm{ult}} b^{3} \sqrt{\mathrm{TOW} Z \mathrm{ZFW}}(1+2 \mathrm{~A})}{(t / c)_{\mathrm{avc}} \cos ^{2} \Lambda_{c t t} S_{W_{G}}^{2}(1+\mathrm{A})} \times 10^{-6}
$$

In this correlation, $n_{\text {utt }}$ is the ultimate load factor, $b$ is the wingspan in feet, $\mathrm{A}$ is the trapezoidal wing taper ratio, $(t / c)_{\text {ave }}$ is the average thickness-to-chord ratio, $\mathbf{A}$, , is the sweep of the wing elastic axis, and $S_{W_{c i}}$ is the gross wing area in square feet. TOW and ZFW are maximum takeoff and zero fuel weights, respectively, in pounds.

\section{Drag}

The induced drag is calculated from the vorticity distribution and induced velocities in the Trefftz plane using

$$
D_{i}=\int_{\text {wake }} \frac{\rho}{2} \boldsymbol{V}_{\text {Trefftz }} \cdot \hat{\boldsymbol{n}} \Gamma(s) \mathrm{d} s
$$

where $\boldsymbol{V}_{\text {Irettz }}$ represents the induced velocities, and $\hat{\boldsymbol{n}}$ the unit normal to the wake in the Trefftz plane.

Profile and compressibility drag are evaluated at panels using section characteristics determined from experimental data. Total drag is obtained by integrating over the configuration.

Profile viscous drag is calculated assuming a quadratic variation with the local section lift coefficient:

$$
c_{\mathrm{dp}}=c_{d 0}+c_{d 2} c_{l}^{2}
$$

$c_{\mathrm{dp}}$ is the section profile drag coefficient, and the coefficients $c_{d / 1}$ and $c_{d 2}$ are estimated based on the method of Ref. 13:

$$
\begin{aligned}
& c_{d 01}=2.04 K_{\mathrm{dp}} c_{j} \\
& c_{d 2}=\frac{0.38}{\cos ^{2} \Lambda} c_{d 0}
\end{aligned}
$$

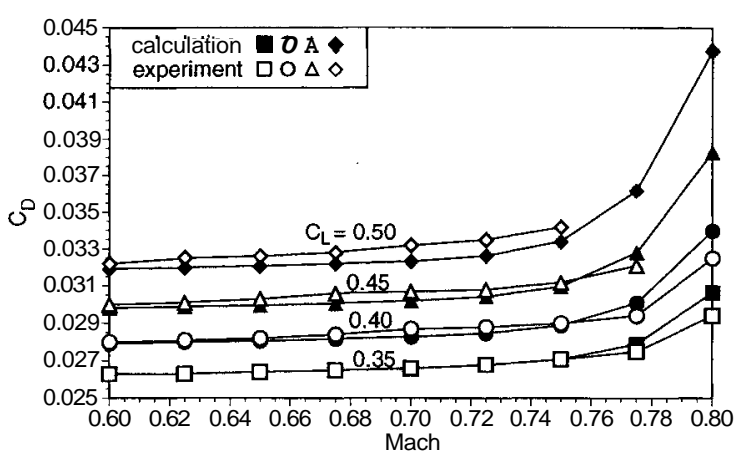

Fig. 4 Comparison of computed and experimental drag values for the DC-9-30.

The skin friction coefficient $c_{f}$ is for a turbulent flat plate at flight Reynolds number. The form factor $K_{\mathrm{dp}}$ is given in Ref. 13. The 2.04 is a correction for wetted area; the 0.38 is based upon an empirical correlation for aircraft drag.

It was found that it is necessary to model the variation of $c_{d t}$ with $t / c$. Since parasite drag is a large component of the total drag, changes in $c_{d 0}$ caused by varying $t / c$ are a consideration that acts to limit maximum section thicknesses.

Compressibility drag is computed by the crest-critical Mach number method described in Refs. 10,13, and 14. This method first determines the Mach number at which sonic flow first appears at the highest point on the airfoil, the crest critical Mach number $M_{\mathrm{cc}}$ :

$$
M_{\mathrm{cc}}=M_{\mathrm{cc}}(\Lambda, t / c, c,)
$$

Compressibility drag is then estimated through a correlation with $M / M_{\mathrm{cc}}$ that is based on flight test data from several commercial transports ${ }^{14}$ :

$$
c_{d_{\mathrm{ppr}}}=c_{d_{\mathrm{cpr}}}\left(M / M_{\mathrm{cc}}, \Lambda\right)
$$

The drag method can generate reasonable estimates, as shown in Fig. 4, which is taken from Ref. IO. The computed values in the figure include nonwing and surface roughness drag components. These contributions are not included for the wing-alone planform optimization results presented in subsequent sections.

\section{Maximum Lift}

A critical section approach is used for predicting wing $C_{L_{\max }}$ in which the distribution of section $c_{l}$ is calculated and compared with the local $c_{l_{\text {max }}}$ at each spanwise section. The wing is said to be at $C_{L_{\max }}$ when the lift coefficient on any section reaches its local $c_{l_{\max }}$.

The critical section method gives surprisingly good results, probably because of the requirement to maintain good handling qualities at stall. Stall should not begin at the wingtips since this could cause undesirable pitch-up or roll. In practice, because of aft sweep, a conventional transport wing is prone to stall at the wingtips, so that aircraft designers modify airfoil sections on the inboard sections to degrade the $c_{l_{\max }}$ of those sections and ensure stall beginning near the wing root. Some margin is provided against stalling the tip sections, so that the wing is designed to stall just below the point where the critical outer section reaches its $c_{I_{\max }}$.

The specific procedure used here compares the streamwise $c_{l}$ calculated from the three-dimensional Weissinger model with the two-dimensional $c_{I_{\max }}$ of the streamwise section. Variation of clean and flapped $c_{l_{\max }}$ with sweep is not included in the present results, based upon experimental observations that values of section $c_{l_{\operatorname{mix}}}$ on swept wings will approach twodimensional values when devices for boundary-layer control, such as fences and vortex generators, are properly applied to the wing. ${ }^{15}$ 


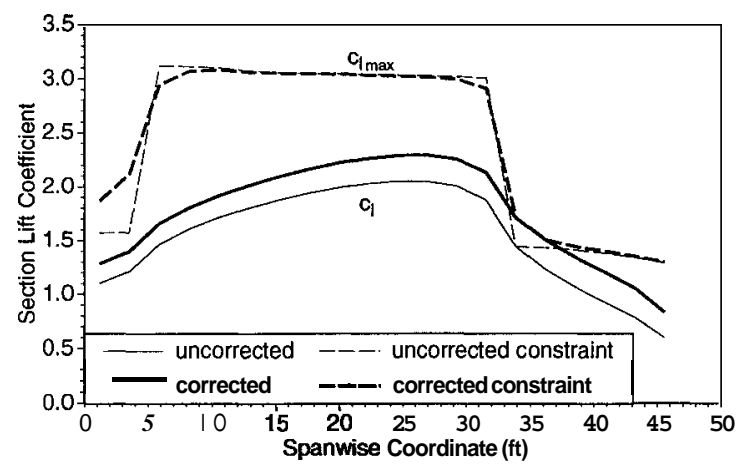

Fig. 5 Section lift coefficient distributions for the DC-9 at maximum lift, with and without corrections for induced camber at the flap edge.

To simulate a wing with flaps deployed, increases are made to section $c_{1}$ at zero angle of attack and $c_{l_{\max }}$. We note these changes with Ac, and $\Delta c_{I_{\text {max }}}$, respectively:

$$
\Delta c_{l}=\Delta c_{l}(\delta) \text { and } \Delta c_{l_{\max }}=\Delta c_{l_{\max }}(\delta)
$$

Ac, and $\Delta c_{l_{\text {max }}}$ are functions of flap deflection 6 , and partially reflect viscous effects. In the three-dimensional Weissinger model, incidences are incremented an amount $\Delta \varepsilon$ that gives a lift increment equivalent to that generated by the flap:

$$
\mathrm{AB}=\Delta c_{l} / c_{l_{\mathrm{u}}}
$$

where $c_{t_{i s}}$ is the section lift curve slope. This $A \boldsymbol{B}$ is applied to the streamwise airfoil section and the Weissinger model predicts the effects due to sweep. Calculation of section stall is done as with unflapped sections except that $\Delta c_{l_{\max }}$ is added to the section $c_{l_{\text {max }}}$.

To avoid underprediction of $C_{L_{\operatorname{mix}}}$, a correction is needed for induced camber near the flap edge. The flap induces upwash on the sections just outboard of the flap edge, increasing section lift. This upwash has a chordwise variation that alters the effective camber and increases the $c_{I_{\text {max }}}$ of these sections. If this increase in $c_{t_{\text {mix }}}$ is unaccounted for, the critical section approach underpredicts $C_{L_{\text {max }}}$ of wings with flaps deployed as indicated in Fig. 5.

To incorporate this effect, estimates of induced flap deflection are made using a method developed in Ref. 10. This method accounts for induced velocities due to the vorticity shed at the flapedge. It assumes a functional fit for the spatial variation of induced velocity, with fit coefficients determined by comparison with calculations of the induced velocities for representative geometries. The function provides an effective induced flap deflection:

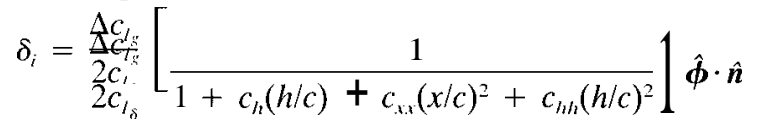

Here, Ac, is the increment in two-dimensional section $c_{l}$ due to geometric flap deflection. It indicates the strength of the vorticity trailing from the flap edge. $c_{l_{\delta}}$ is the two-dimensional flap lift curve slope used to convert $c_{l}$ to flap deflection. $\mathbf{x}$ and $h$ are spatial factors. They are components of a position vector between three-quarter chord points of the flap edge section and any section. $\mathbf{x}$ gives the distance in the $\hat{x}$ direction; $h$ gives the distance perpendicular to the $\hat{\boldsymbol{x}}$ direction: $h$ $=\sqrt{y^{2}+z^{2}}$. The dot product, $\boldsymbol{\phi} \cdot \hat{\boldsymbol{n}}$, gives 6 , the correct sign according to the direction of induced velocity $\phi$ and the local panel normal $\hat{\boldsymbol{n}}$. The induced flap angle changes from negative to positive when moving from the flapped to unflapped wing sections. The coefficients $c_{l l}, c_{x x}$, and $c_{l_{l} l}$, are fit constants determined from a study presented in Ref. 10:

$$
\mathrm{c},=13, \quad c_{x a}=151, \quad c_{h h}=18
$$

The induced flap deflection 6 , is added to the geometric deflection $\delta_{g}$ to obtain the effective flap deflection 6 , used to determine section $c_{I_{\max }}$.

The critical section analysis with correction for induced flap incidence was applied within the wing optimization program to evaluate flaps down $C_{L_{\text {mix }}}$ for the DC-9 and DC-10. Basic section $C_{L_{\text {max }}}$ was estimated using data from Ref. 16 and internal information on DC-9 and DC-10 airfoil families (Fig. 6). Increments to section lift $\Delta c_{l}$ and $\Delta c_{l_{\max }}$ were estimated using methods suggested by Ref. 17 (Fig. 7 ). The resulting aircraft $C_{L_{\text {max }}}$ is plotted as a function of flap deflection in Fig. 8 , where flight test data from Ref. 13 is also provided for comparison. The data were corrected for 1-g flight from certification values, and section $c_{l_{\max }}$ was reduced by 0.237 to provide a margin against tip stall. ${ }^{10}$

A final adjustment to $c_{l_{\max }}$, due to slats of $1.0 \cos ^{2} \Lambda$ is made, where 1.0 is an estimated increment in two-dimensional $c_{l_{\max }}$ due to slats, and $\cos ^{2} \Lambda$ is an estimate of the variation with sweep made from data in Ref. 18.

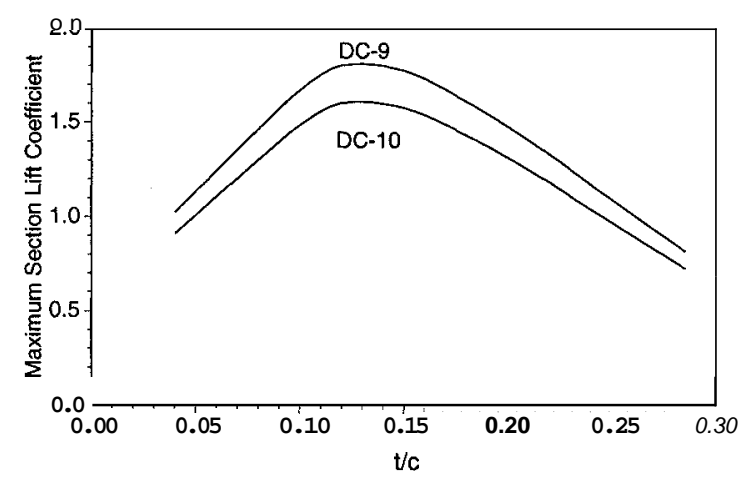

Fig. 6 Model for maximum section lift coefficient as a function of $d c$.

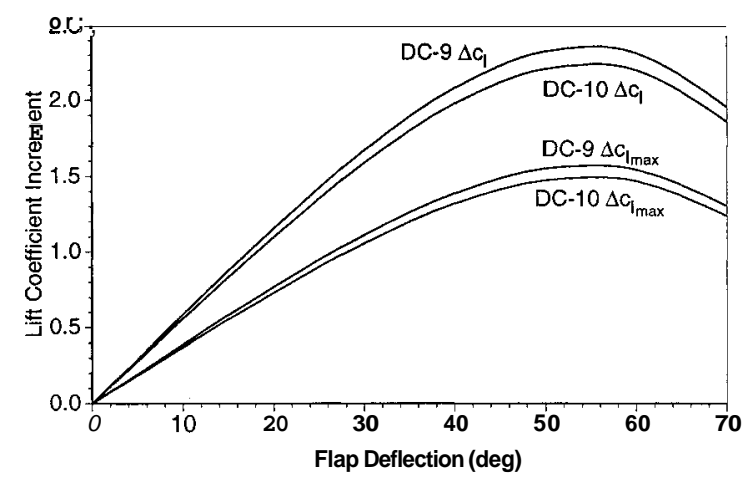

Fig. 7 Model for the increment in section lift due to flap deflection $\Delta c_{l}$, and the increment in maximum section lift $\Delta c_{l_{\max }}$.

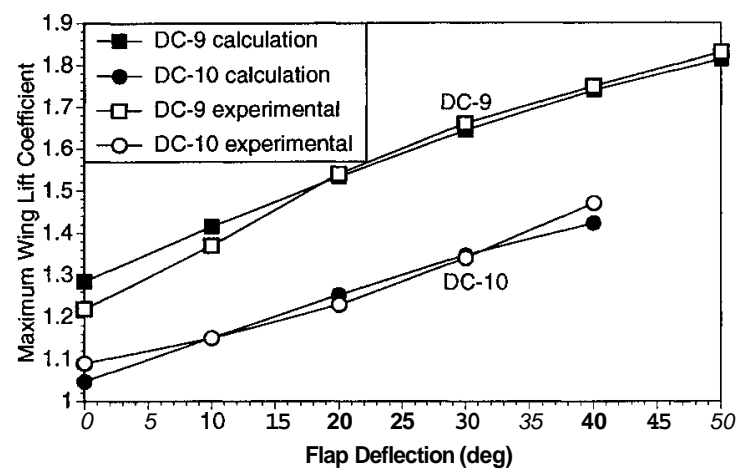

Fig. 8 Maximum lift calculated by the wing optimization program using the $\delta_{i}$ correction are compared to flight test data for DC-9 and DC-10 aircraft, slats retracted. 
Table 1 Design variables and constraints for the DC-9 baseline and the optimized designs

\begin{tabular}{|c|c|c|c|c|c|c|c|c|c|}
\hline True & Variable & Units & $\%$ b12 & Base & $\mathbf{A}$ & $\mathrm{B}$ & $\mathrm{C}$ & $\mathrm{D}$ & $\mathbf{E}$ \\
\hline Objective & $D_{\text {wing }} / D_{\text {winghasi }}$ & & & & 0.78 & 0.90 & 0.96 & 0.94 & $0.9 \mathrm{I}$ \\
\hline \multirow[t]{5}{*}{ Constraint } & $m_{\text {wing }} / 2$ & $\mathrm{lb}$ & & & 5,815 & 5,815 & 5,815 & 5,621 & 4,897 \\
\hline & $m_{\text {fucl }} / 2$ & $\mathrm{lb}$ & & & & & & 11,839 & 11,839 \\
\hline & Fuel fraction & & & 0.89 & & & & 1.00 & 1.00 \\
\hline & $\begin{array}{r}\text { Climb } c_{l}-c_{l \max } \\
20 \text { constraints }\end{array}$ & & & & & & $J$ & $J$ & $J$ \\
\hline & $\begin{array}{l}\text { Landing } c_{l}-c_{l \max } \\
20 \text { constraints }\end{array}$ & & & & & & $J$ & $J$ & $J$ \\
\hline \multirow[t]{19}{*}{ Design } & $S / 2$ & $\mathrm{ft}^{\prime}$ & & 501 & 406 & 349 & 465 & 452 & 445 \\
\hline & $\mathrm{bl}(2 \cos \phi)$ & $\mathrm{ft}$ & & 46.6 & 54.5 & 49.2 & 47.9 & 47.9 & 46.9 \\
\hline & $c / c_{\mathrm{row}}$ & & 30 & 0.761 & 0.693 & 0.672 & 0.629 & 0.652 & 0.617 \\
\hline & & & 70 & 0.442 & 0.174 & 0.275 & $0.41 \mathrm{I}$ & 0.479 & 0.493 \\
\hline & & & 90 & 0.284 & 0.028 & 0.084 & 0.238 & 0.282 & 0.278 \\
\hline & & & 100 & 0.204 & 0.010 & 0.011 & 0.109 & 0.131 & 0.129 \\
\hline & $\mathbf{A}$ & $\operatorname{deg}$ & & 24.5 & -1.8 & 32.8 & 29.6 & 30.9 & 31.2 \\
\hline & $\&$ & $\mathrm{deg}$ & 0 & 0.0 & -0.7 & 0.1 & 0.3 & 0.3 & 0.2 \\
\hline & & & $10^{\mathrm{b}}$ & 0.0 & 0.0 & 0.0 & 0.0 & 0.0 & 0.0 \\
\hline & & & 30 & -0.2 & -0.1 & -0.4 & 0.3 & $0 . \mathrm{I}$ & 0.7 \\
\hline & & & 70 & -1.4 & 2.5 & 0.0 & -1.0 & -1.5 & -0.2 \\
\hline & & & 90 & -3.0 & 8.5 & 0.2 & -2.6 & -3.0 & -1.4 \\
\hline & & & 100 & -3.0 & 9.7 & 0.7 & -3.3 & -3.8 & -1.7 \\
\hline & tic & & 0 & 0.131 & & 0.148 & 0.146 & 0.143 & 0.149 \\
\hline & & & 30 & 0.109 & & 0.125 & 0.123 & 0.123 & 0.122 \\
\hline & & & 70 & 0.102 & & 0.079 & $0 . \mathrm{I} 15$ & 0.118 & 0.123 \\
\hline & & & 90 & 0.093 & & 0.034 & 0.119 & 0.121 & 0.125 \\
\hline & & & 100 & 0.083 & & 0.010 & 0.118 & 0.119 & 0.123 \\
\hline & Fuel fraction & & & 0.89 & & & & 1.00 & 1.00 \\
\hline
\end{tabular}

"Wing mass constraints vary depending on the structural considerations assessed and match the mass of the baseline under the same analysis.

${ }^{n}$ The incidence at $10 \%$ semispan is fixed at zero

\section{Optimization}

Designs are refined with a gradient-based optimizer that uses a sequential quadratic programming algorithm as described in Ref. 19. For the results in this article, drag was minimized at fixed weight and with maximum constraints on section lift coefficient. Several objective functions were investigated including minimizing a combination of drag and weight, $D+K W$, and maximizing range calculated through the Breguet range formula.

While these objectives yield successful results, the fixed weight objective was selected for simplicity. With wing weight fixed, minimizing drag increases range. This objective also constrains airframe cost, which varies with weight, and causes reductions in total cost entirely through reductions in fuel cost. This objective achieves the desired results while representing relative importance of drag vs weight implicitly by the selected value of wing weight.

Design variables and constraints are listed in Table 1. Chord, twist, and $t / c$ are varied along the span of the wing. The fuel fraction variable represents the fraction of available fuel volume actually occupied. It sets the fuel weight and is constrained to be less than or equal to one. A separate section lift constraint is used for every panel; using a single constraint that indicates the worst violation of any panel can prevent convergence because the single composite constraint is nonsmooth at points where the worst violation switches from one panel to another. The sequential quadratic programming algorithm is amenable to large numbers of constraints: using multiple constraints instead of a single composite adds no extra work in function evaluations for gradient calculations, and it gives the optimizer better information for navigating the design space.

\section{Results}

To demonstrate the effects of different constraints on optimal design, a series of wings was optimized from a DC-932 planform using increasingly realistic considerations. The results are shown in Fig. 9 and Table 1.

In the analysis, drag is evaluated at Mach $0.75,30,000 \mathrm{ft}$ altitude, and an aircraft weight of 108,000lb. Structural weight is examined at Mach $0.84,25,000 \mathrm{ft}$ altitude, and aircraft weights of $108,000 \mathrm{lb}$ with wing fuel and $87,000 \mathrm{lb}$ without fuel. Maximum lift conditions are evaluated at sea level, 135 $\mathrm{kn}$ slats down and $117 \mathrm{kn}$ slats and flaps down.

The first design, for minimum-induced drag at fixed weight, is strongly dominated by structural considerations. Since only induced drag is considered, the optimal design needs a large span with a good cruise lift distribution. The lift distribution is set using wing incidence angles. The large span is achieved by making a highly tapered planform. For this case, $t / c$ was not optimized because there are no upper limits on $t / c$ unless parasite drag, compressibility drag, or high lift are considered. The depth of the structural box is then tied to the local chord length. The highly tapered planform improves structural efficiency by making the structural box very deep near the wing root, where bending moments are large. It also limits the load that is added onto the wingtips between cruise and the critical structural design condition, providing an aerodynamically efficient spanload at cruise, and a structurally efficient spanload at the condition determining wing weight.

When compressibility and profile drag are added, a more conventional planform results. Section thicknesses are optimized, and the introduction of compressibility drag causes the wing to be swept. Unlike the induced drag objective, the total drag objective is sensitive to section lift coefficients. A drag penalty occurs naturally for wings with sections that have excessively high lift coefficients. This, however, is not sufficient to prevent excessive taper, as indicated by the second planform in the figure.

Reasonable planforms can only be attained by imposing constraints on low-speed performance. These constraints require the wing to fly at specified minimum speeds without any section exceeding maximum limits on lift coefficient. The result of applying these constraints is shown by the third planform in the figure. Low-speed constraints are important in defining the planform near the wingtip; they require larger tip chords and thicknesses than would be obtained considering only weight and drag.

The effect of including bending relief due to fuel inertia is shown in the fourth planform. Since fuel is assumed to be evenly distributed according to wing volume, the optimized 


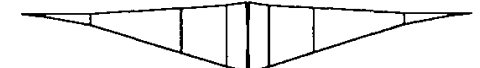

a)

Minimum induced drag at fixed weight.

b)
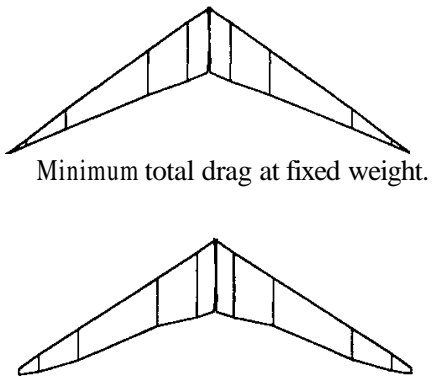

Minimum total drag at fixed weight c) with low speed lift constraints.

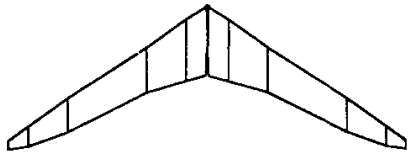

Minimum total drag, fixed weight, low speed lift constraints, and fuel inertia

d) relief.

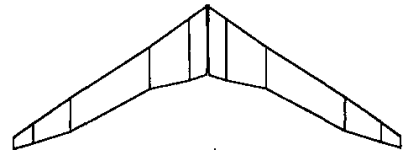

Minimum total drag, fixed weight, low speed lift constraints, fuel inertia

e) relief, and static aeroelasticity.

Fig. 9 Effects of various considerations on optimal design.

design has larger tip chords than the previous case. By distributing fuel toward the wing tips, the wing maximizes the amount of bending relief, improving structural efficiency. Total fuel capacity was kept constant by adjusting the fraction of available fuel volume actually occupied by fuel.

When static aeroelasticity is introduced in the final planform, changes in wing shape are minor, but savings in wing weight are substantial. Aeroelasticity tends to unload the wing tips in structurally critical maneuver or gust conditions. This reduces bending moments in the structurally critical condition and results in a lighter structure for a given cruise spanload. Since wing weight is constrained to equal the weight of the baseline wing evaluated under the same structural considerations, the low baseline weight restricts the aeroelastic design from increasing span or area - changes that might result given an objective that permitted trading weight for drag.

Relative to the baseline, the final planform has more sweep and thickness. The increased $t / c$ nearly maximizes section $c_{l_{\max }}$ as given by Fig. 6 . This results in greater wing $C_{L_{\operatorname{mix}}}$ and permits a smaller wing area, reducing profile drag. The increased sweep is required to limit increases in compressibility drag from the thicker sections. An actual design would need to address many issues that were not included in this optimization. The increase in sweep may be restricted by gear placement and trim issues.

The last three planforms exhibit small chord extensions. Chord extensions are normally considered as a way to ease the integration of gear into the wing; however, the optimized wings have developed chord extensions because of an interesting interaction between compressibility drag and structural weight. The chord extension improves structural box depth at the wing root without increasing $t / c$. This also reduces section lift coefficients at the wing root, reducing the severity of compressibility drag and enabling an increase in root $t / c$, further increasing structural efficiency.

\section{Conclusions}

A study was made of the basic influences of drag, weight, maximum lift, and other considerations on optimal wing planform shape. Aerodynamic and structural analyses were developed to model induced, profile, and compressibility drag, bending and buckling weight, section maximum lift constraints, and static aeroelasticity.

The importance of off-design conditions can be seen in the study results where design considerations were progressively added to the optimization to show the effect of each on optimal wing shape. Induced drag and structural considerations strongly favor highly tapered wings to attain large spans. Parasite and compressibility drag have limited effect on wing taper, making maximum lift constraints necessary for generating realistic tip chords. Only with sufficient breadth of analysis can realistic wing planform shapes be obtained.

Further development is underway to extend the wing optimization method to wing/tail combinations. In its current state, this method is a strong tool for preliminary design studies of new configurations; it forms a basis for rational comparison of configurations such as forward swept wings and a variety of wingtip devices.

\section{References}

'Mason, W., "Analytic Models for Technology Integration in Aircraft Design," AIAA Paper 90-3262, Sept. 1990.

'Grossman, B., Haftka, R., Kao, P., Polen, D., Rais-Rohani, M., and Sobieszczanski-Sobieski, J., "Integrated Aerodynamic-Structural Design of a Transport Wing," Journal of Aircraft, Vol. 27, No. 12, 1990, pp. 1050-1056.

${ }^{3}$ Kroo, I., "A General Approach to Multiple Lifting Surface Design and Analysis," AIAA Paper 84-2507, Nov. 1984.

'Craig, A., and McLean, J., "Spanload Optimization for Strength Designed Lifting Surfaces," AIAA Paper 88-2512, June 1988.

'McGeer, T., "Wing Design for Minimum Drag with Practical Constraints," Ph.D. Dissertation, Stanford Univ., Dept. of Aeronautics and Astronautics, Stanford, CA, Dec. 1983.

"McGeer, T., "Wing Design for Minimum Drag with Practical Constraints," Journal of Aircraft, Vol. 21, No. 11, 1984, pp. 879886.

${ }^{7}$ Hutchison, M., Unger, E., Mason, W., Grossman, B., and Haftka, R., "Variable-Complexity Aerodynamic Optimization of a High-speed Civil Transport Wing," Journal of Aircraft, Vol. 31, No. 1, 1994, pp. $110-116$.

"Gallman, J., Kroo, I., and Smith, S., "Optimization of JoinedWing Aircraft," Journal of Aircraft, Vol. 30, No. 6, 1993, pp. 897905.

"Wakayama, S., and Kroo, I., "A Method for Lifting Surface Design Using Nonlinear Optimization,” AIAA Paper 90-3290, Sept. 1990 .

"'Wakayama,S, "Lifting Surface Design Using Multidisciplinary Optimization," Ph.D. Dissertation, Stanford Univ., Dept. of Aeronautics and Astronautics, Stanford, CA, Dec. 1994.

"Megson, T., Aircraft Structures for Engineering Students, 2nd ed., Halsted Press, New York, 1990, Sections 8.1, 8.4, and 8.5.

"Gray, W., and Schenk, K., "A Method for Calculating the Subsonic Steady-State Loading on an Airplane with a Wing of Arbitrary Planform and Stiffness," NACA TN 3030, Dec. 1953.

"Shevell, R. S., Fundamentals of Flight, 2nd ed., Prentice-Hall, Englewood Cliffs, NJ, 1989, Chaps. 11, 12, and 18.

${ }^{1+}$ McGeer, T., and Shevell, R., "A Method for Estimating the Compressibility Drag of an Airplane," Stanford Univ., Dept. of Aeronautics and Astronautics, SUDAAR 535, Stanford, CA, Jan. 1983.

"Furlong, Chester, G., and McHugh, J. G., "A Summary and Analysis of the Low Speed Longitudinal Characteristics of Swept Wings at High Reynolds Number," NACA Rept. 1339, May 1957.

"Abbot, I., and Van Doenhoff, A., Theory of Wing Sections, Dover, New York, 1959, Sections 7.4 and 8.5.

"Torenbeek, E., Synthesis of Subsonic Airplane Design, Delft Univ. Press. Delft, Holland, 1982, Appendix G.

"Callaghan, J. G., "Aerodynamic Prediction for Aircraft at Low Speeds with Mechanical High Lift Devices," AGARD Lecture Series 67, von Karman Inst., Brussels, Belgium, May 1974.

"Gill, P. E., Murray, W., and Wright, M. H., Practical Optimization, Academic, New York, 1981, Sections 6.4.1, 6.5.3, and 6.5.5. 